\title{
BACKSCATTERING ANALYSIS OF OFFSHORE PLATFORMS IN GULF OF MEXICO VIA MULTI-POLARIZATION TERRASAR-X/TANDEM-X DATA
}

\author{
Domenico Velotto ${ }^{1}$, Armando Marino ${ }^{2}$ and Ferdinando Nunziata ${ }^{3}$ \\ ${ }^{1}$ DLR, German Aerospace Center, Remote Sensing Technology Institute \\ 28199 Bremen, Germany \\ ${ }^{2}$ The Open University, School of Engineering and Innovation \\ MK7 6AA Milton Keynes, U.K. \\ ${ }^{3}$ Università Parthenope, Dipartimento di Ingegneria \\ 80133 Napoli, Italy
}

\begin{abstract}
Satellite-based synthetic aperture radar (SAR) has been proven to be an effective tool for maritime safety and security. In this framework, monitoring oil and gas offshore platforms is a key topic taken into account the high risk of accident, e.g. exposed to extreme weather conditions, and the potential threats to the environment, e.g. release of polluting material into the ocean.

In this study, offshore platform monitoring is discussed using multi-polarization X-band SAR imagery. For operational purposes, the analysis is undertaken using a data set of dual-polarization TerraSAR-X/TanDEM-X (TS-X/TD-X) imagery collected over a test site in Gulf of Mexico at low and high incidence angles. Additionally, experimental Dual Receive Antenna (DRA) bistatic quadpolarization TD-X data will be used for a more in depth analysis of the backscattering properties and detection performance of different target polarimetric detectors.

The motivation behind this work is the observation that, under low incidence angle and moderate wind conditions, co-polarized channels may fail in detecting offshore platforms even when fineresolution imagery is considered. The multi-temporal dataset allows investigating the possible causes of this unexpected behavior and to draw some conclusions on the target's backscattering depending on polarization, resolution and incidence angle.
\end{abstract}

Index Terms - SAR, polarimetry, radar, target detection, oil platform

\section{INTRODUCTION}

The recent discovery of a significant number of oil and gas deposits in the seabed increased the amount of offshore platforms worldwide [1]. Moreover, with the advent of deep water drilling technology, their installation is not fixed to the seabed and their location is not limited to coastal water anymore. Conventional surveillance systems, like coastal-based radars, flight surveys or patrol control can provide abundant information on platforms locations and operational status, but only with limited spatial and temporal coverage and at a high cost for equipment and manpower.

The Gulf of Mexico is one of the most important regions for energy resources and infrastructure in the world. Besides disaster event such as the Deepwater Horizon platform blowout, there are thousands of minor spills every year. Figure 1 shows the known positions of the offshore platform (white rectangles)

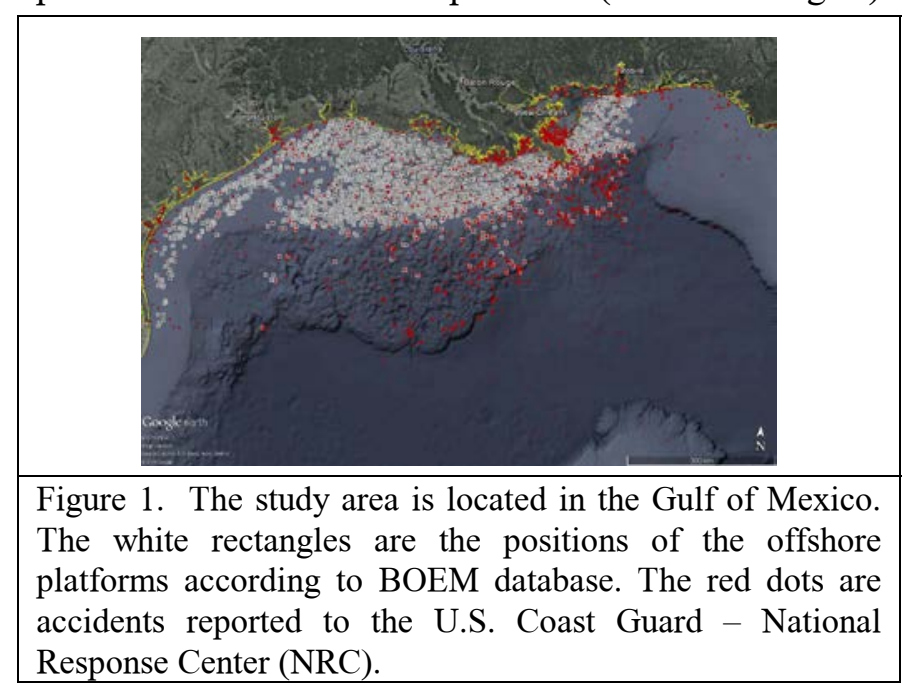


and accidents in the same regions (red dots). In the period $2010-2015$, about $70 \%$ of the accident are oil spills, of which about $65 \%$ caused by offshore platforms.

Satellite-based synthetic aperture radar (SAR) has been proven to be an effective tool for maritime safety and security because of radar almost all-weather and all-day acquisition capabilities [2]. Monitoring offshore platforms is necessary, as shown in [3] and [4] concerning the ocean pollution, but needed as well for the safety of ship traffic. The detection and localization of metallic targets over the ocean surface by means of a radar sensor is quite straightforward. However, here it is shown that the information provided by backscattered intensity collected by a single-polarization SAR it is not always sufficient for this task. By means of dual-polarization SAR data acquired by the satellites TerraSAR-X/TanDEM-X (TS-X/TD-X) the unexpected backscattering behavior of offshore platform under different polarization, resolution and incidence angle conditions is explained.

\section{DATA SETS}

In this study, offshore platform monitoring is discussed using multi-polarization high resolution Xband SAR imagery. For operational purposes, the analysis is undertaken using a data set of dualpolarization TS-X/TD-X imagery collected over a test site in Gulf of Mexico at low and high incidence angles. The TS-X/TD-X dataset has been collected in all possible dual-polarization combinations at two different viewing geometries using repeat pass acquisitions. All products have been acquired during satellite descending orbit (right looking) in StripMap (SM) mode which provides a nominal spatial resolution of $1.2 \mathrm{~m} \times 6.6 \mathrm{~m}$ (range $\mathrm{x}$ azimuth) and the L1b SLC data format is processed. Additionally, experimental Dual Receive Antenna (DRA) bistatic quad-polarization TD-X data will be used for a more in depth analysis of the backscattering properties and detection performance of different target polarimetric detectors.

To gain additional information on the offshore platform structure, location and type, free-cloud multispectral Sentinel-2 Multi-Spectral Instrument (MSI) and Landsat8 Operational Land Imager (OLI) are used in combination with the Bureau of Ocean Energy Management (BOEM) database. Figure 2 shows a Region of Interest (ROI) in the Gulf of Mexico as

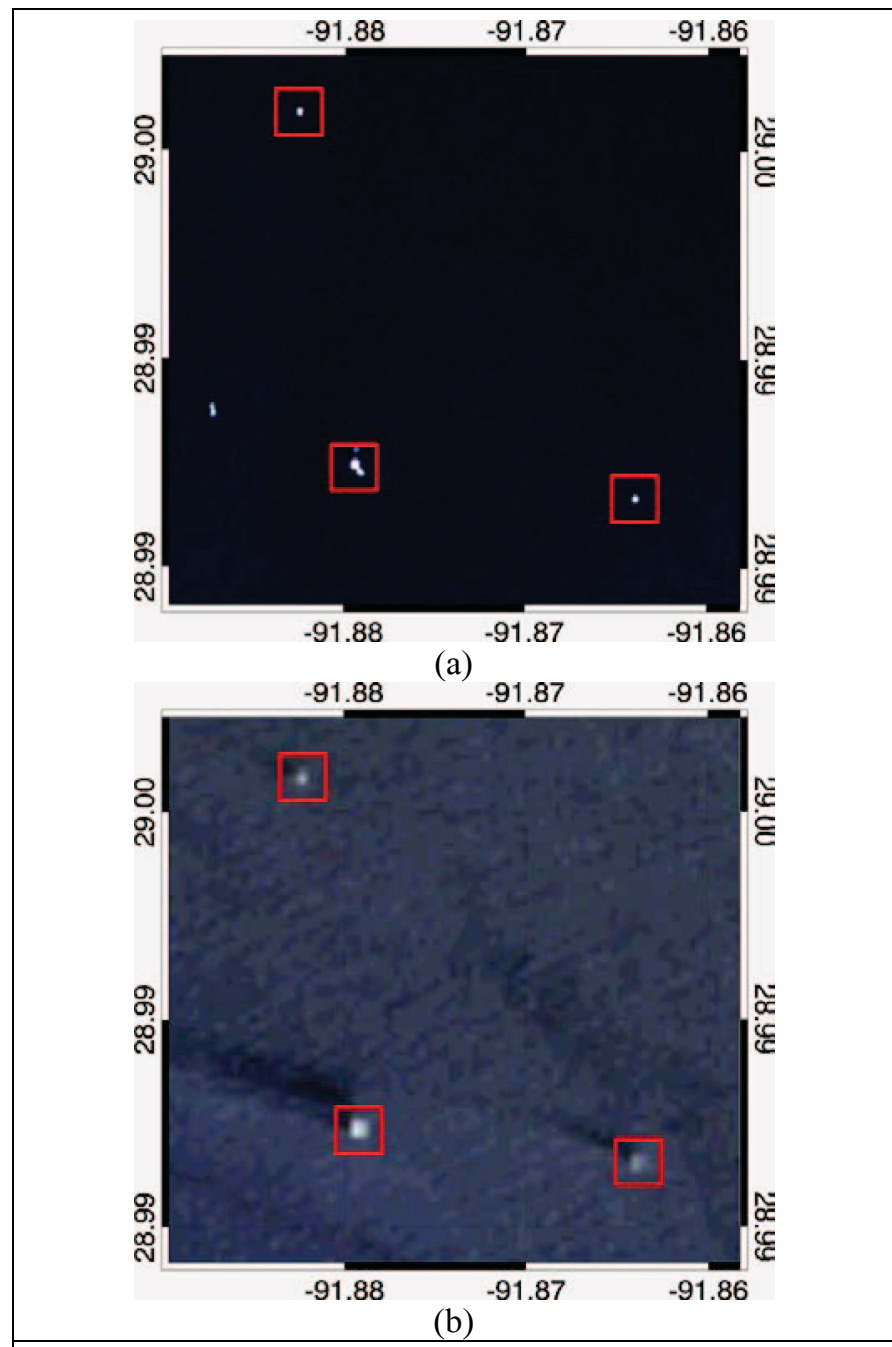

Figure 2. Optical visible bands (a) Sentinel-2 $10 \mathrm{~m}$ resolution (b) Landsat8 OLCI 30m

imaged by the Sentinel-2 (a) and Landsat8 (b) using the red, green and blue visible bands. Three platforms could be identified and are indicated with red rectangles. Please note that due to the higher resolution (10m) of Sentinel-2 (Figure 2a) in comparison of the Landsat8 (Figure 2b) more details on the objects' structure and size are deductible. We are talking of three metal structures with size greater than $30 \mathrm{~m}$.

\section{PLATFORM BACKSCATTERING}

To analyze the backscattering behavior of the offshore platforms under different linear transmit/receive polarizations and with respect to the incidence angle, the dual-polarimetric $\mathrm{HH}-\mathrm{VV}$ combination is considered. 


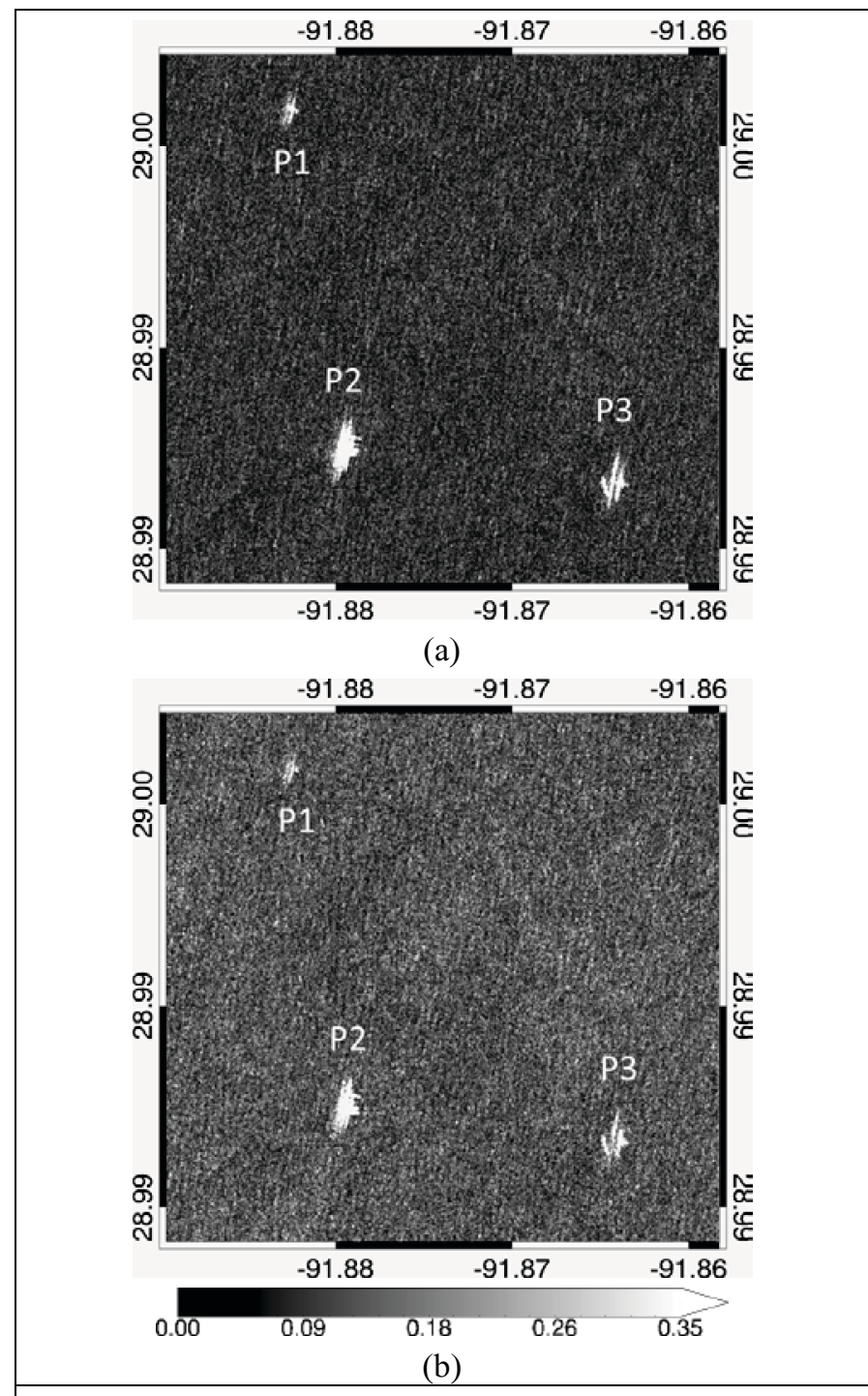

Figure 3. Ground projected calibrated amplitude SAR data collected at high incidence angle by TS-X/TD-X over a cluster of 3 offshore platforms in GoM (labeled as P1, P2 and P3). (a) the co-pol channel HH; (b) the co-pol channel VV.

Platforms installed in shallow water consist of vertical metallic towers sustained by submersed pylons fixed to the sea floor. The tower's altitude can be of several tens of meters and hence it may cause several scattering mechanisms caused by the different radar propagation and reflection paths that result in multiple bright spots aligned along the range direction. These signatures are evident in the high resolution $\mathrm{X}$ band SAR data shown in Figure 3, where both co-pol channel are displayed, i.e. HH in Figure $3 \mathrm{a}$ and VV in Figure $3 b$. However, one can note that the radar backscatter of such big targets reduces significantly

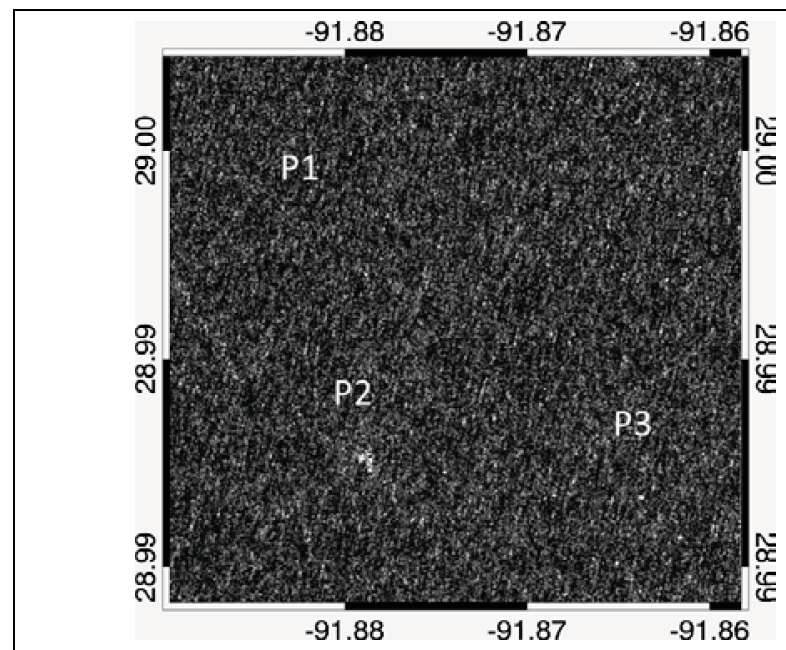

(a)

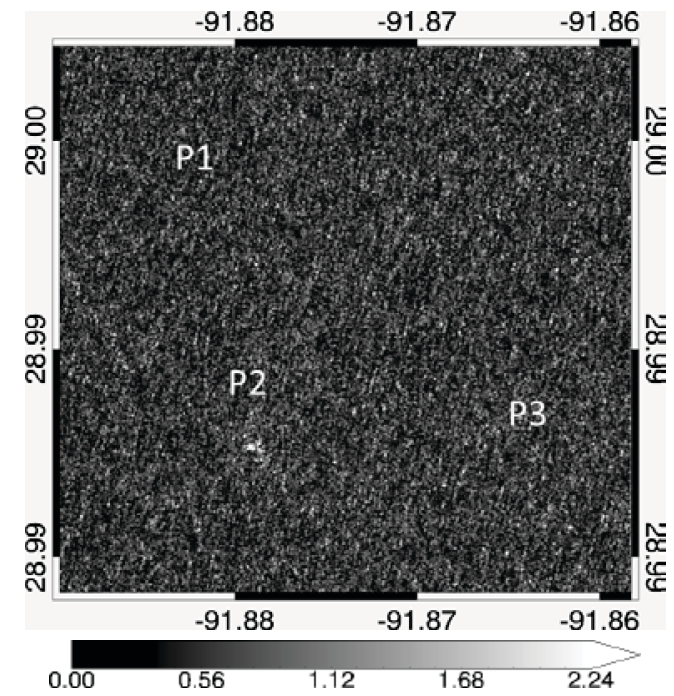

(b)

Figure 4. Ground projected calibrated amplitude SAR data collected at low incidence angle by TS-X/TD-X over a cluster of 3 offshore platforms in GoM (labeled as P1, P2 and P3). (a) the co-pol channel HH; (b) the co-pol channel VV.

(apparently it vanishes) in both co-pol channels of the dataset collected with low incidence angle (see Figure $4 a$ and $b)$.

\section{PRELIMINARY RESULTS}

In this section, a physical explanation of the odd results provided by co-polarized imagery collected at low incidence angles will be provided. Polarimetric decomposition techniques will be applied to either HH-VV dual-polarization and quad-polarization dataset. A preliminary analysis of the Pauli 
decomposition shows that dihedral scattering plays an important role in platform's backscattering.

\section{REFERENCES}

[1] "International Energy Agency." [Online]. Available: http://www.iea.org/. [Accessed: 18-Apr-2016].

[2] J. C. Curlander and R. N. McDonough, Synthetic Aperture Radar: Systems and Signal Processing. Wiley, 1991.

[3] X. M. Li, T. Jia, and D. Velotto, "Spatial and Temporal Variations of Oil Spills in the North Sea Observed by the Satellite Constellation of TerraSAR-X and TanDEM-X," IEEE J. Sel. Top. Appl. Earth Obs. Remote Sens., vol. 9, no. 11, pp. 4941-4947, Nov. 2016.

[4] S. Singha, R. Ressel, D. Velotto, and S. Lehner, "A Combination of Traditional and Polarimetric Features for Oil Spill Detection Using TerraSAR-X," IEEE J. Sel. Top. Appl. Earth Obs. Remote Sens., vol. 9, no. 11, pp. 4979-4990, Nov. 2016. 